\title{
The best drug treatment for psychotic depression: antidepressants, antipsychotics or both combined? COMMENTARY ON... COCHRANE CORNER ${ }^{\dagger}$
}

\author{
Katharine Smith
}

\begin{abstract}
SUMMARY
Psychotic depression is a severe condition. Drug treatment (antipsychotics, antidepressants or the combination) or electroconvulsive therapy (ECT) are both effective. However, a 2005 Cochrane review of drug treatments could not find a clear benefit for combination treatment with antidepressant and antipsychotic medication over antidepressants alone. The current updated Cochrane review (2015) incorporated two larger studies and found evidence of superiority for combination treatment. The review was constrained by the small number of available studies and could not address key questions such as the relative merits of antipsychotics and/or antidepressants compared with ECT or ketamine in acute treatment, and which treatments are best for long-term maintenance and prevention of recurrence. However, the methodology used was rigorous and the review gives the best summary to date of the evidence, as well as providing a platform for informing future research.
\end{abstract}

\section{DECLARATION OF INTEREST}

None

\section{Clinical setting}

Depression is a common psychiatric disorder with significant associated disability (Kessler 2003). Within the broad category of depression, psychotic depression is a recognised subtype. Defined as a depressive episode with psychotic features (i.e. delusions and/or hallucinations) in the context of a major depressive disorder, it is a severe condition with a high risk of relapse and recurrence (Rothschild 2003).

Psychotic depression is more common than might at first be assumed, but estimates vary somewhat among different countries and different settings. In the US Epidemiologic Catchment Area Study, the lifetime prevalence of psychotic symptoms in individuals who had ever met the criteria for major depression was $14 \%$, and the lifetime prevalence of psychotic depression was $0.6 \%$ (Johnson 1991). In a European general population study, $18.5 \%$ of respondents with a major depressive episode had psychotic features; the prevalence of psychotic depression was $0.4 \%$ and of nonpsychotic depression 2.0\% (Ohayon 2002). Data on in-patients come from an older study: in a US study of in-patients with major depression, 25\% met the criteria for psychotic depression (Coryell 1984). Compared with non-psychotic depression, psychotic depression is associated with greater severity, increased incapacity, a lower likelihood of placebo response, longer duration of episodes and recurrence of psychotic features in subsequent episodes (Coryell 1998).

\section{Treatment options}

Both pharmacotherapy and electroconvulsive therapy (ECT) are recommended as first-line treatments for psychotic depression (National Institute for Health and Care Excellence (NICE) 2009; American Psychiatric Association 2010; Cleare 2015), and where pharmacotherapy is used, these guidelines recommend a combination of an antidepressant and an antipsychotic. However, there is ongoing discussion about whether the combination is better than monotherapy alone (Wijkstra 2007), particularly as the previous edition (Wijkstra 2005) of this month's Cochrane Corner review (Wijkstra 2015) could not find convincing evidence in favour of combination treatment over antidepressants alone.

The traditional view is that antidepressants treat depressive symptoms and antipsychotics treat psychotic symptoms, implying that a combination of both drugs is needed to treat psychotic depression effectively. An alternative view is that psychotic depression is at the severe end of a continuum of depressive illness, implying that adequate antidepressant treatment alone might
ROUND THE CORNER
Katharine Smith is an honorary consultant psychiatrist at the National Institute for Health Research (NIHR) Oxford cognitive health Clinical Research Facility and Oxford University Department of Psychiatry.

Correspondence Dr Katharine Smith, NIHR Oxford cognitive health Clinical Research Facility, Warneford Hospital, Oxford OX3 7JX, UK. Email: katharine.smith@psych.ox.ac.uk

Copyright and usage (C) The Royal College of Psychiatrists 2017.

${ }^{\dagger}$ See p. 2, this issue. 
be equally effective. Similarly, second-generation ('atypical') antipsychotic drugs such as quetiapine and olanzapine have some evidence for efficacy in mood disorders, implying that the possibility of using these antipsychotics alone should also be investigated.

\section{Previous evidence}

The evidence to date has been difficult to interpret. Randomised controlled trials (RCTs) in such a population tend to be small and difficult to perform. Psychotic depression can be a life-threatening disorder, so patients may need to be treated more quickly than an RCT of pharmacological therapy might allow. ECT is often preferred in very urgent cases and so randomisation would be problematic. By definition, psychotic depression includes some loss of insight, often with associated anxiety, so it may be difficult to obtain informed consent and recruit patients. Also, given the relative urgency, treatment with placebo, even in the short term as part of a placebo-controlled RCT, raises ethical problems, as the delay in treatment from being included in the placebo arm carries significant risks.

A previous meta-analysis (Parker 1992) did not find a significant difference in treatment effect between tricyclic antidepressants alone or in combination with first-generation ('typical') antipsychotics. However, the results of the meta-analysis were limited by broad inclusion criteria, which meant that the quality of included studies varied a lot. Studies with inadequate methodology and also retrospective, uncontrolled or non-randomised designs were included in the meta-analysis.

In contrast to the review by Parker et al from 1992, both the original 2005 review by Wijkstra et al and its update in 2015 used stricter inclusion criteria demanded by the Cochrane review methodology. This meant that only RCTs were included and that the authors of the review clearly predefined how they would include or exclude data from studies that presented summary data on groups with mixed diagnoses (nonpsychotic/psychotic depression or unipolar/ bipolar depression). Further details are explained in the methods section below. The original 2005 review by Wijkstra et al, using this methodology, had concluded that first-line pharmacological treatment showed equal evidence of efficacy for an antidepressant in combination with an antipsychotic, or an antidepressant alone (but not for an antipsychotic alone). The current review (Wijkstra 2015) aimed to update this review by including new data published subsequently.

\section{Methods}

The authors updated (in April 2013) their previous search for new RCTs of the pharmacological treatment of psychotic depression. Few studies included psychotic depression as a discrete group, so the reviewers searched all RCTs of pharmacological treatment of major depression to identify whether they included a subgroup with psychotic features. Where results for the group with psychotic symptoms were reported (or could be obtained from study authors) these data were included in the review.

Participants of any age and in both out-patient and in-patient settings were included. Psychotic depression was defined as psychotic features (hallucinations and/or delusions) in the context of major depression. Comorbidities with other psychiatric disorders were allowed. Treatment needed to be for a minimum of 4 weeks, with an antidepressant alone, antipsychotic alone or combination of both, compared with each other or placebo. Primary outcomes were 'response' as assessed by a $50 \%$ reduction in score on the Hamilton Rating Scale for Depression (HRSD or HAM-D), or the Montgomery-Åsberg Depression Rating Scale (MADRS) or similar rating scales, and by drop-out rates (as a proxy for acceptability).

Some trials also included patients with a diagnosis of bipolar depression, so that the patient group was a mixture of those with bipolar depression and those with unipolar depression. The reviewers decided that if these results could not be separated out according to diagnosis, the whole trial would be included in the analysis of the review, as long as the number of participants with bipolar depression was $20 \%$ or less of the total number. This is a pragmatic strategy that has been implemented in a number of Cochrane reviews where diagnostic groups could not be separated and it ensures that trials can be included as long as the majority of participants meet criteria.

\section{Results of the review}

In the search performed for the 2005 review, 10 RCTs (a total of 548 participants) were identified (Wijkstra 2005). In the updated search of 2013, 2 additional larger studies were identified, giving a total of 12 studies and 929 participants. The studies varied in methodology. All used procedures to confirm diagnoses, but these varied from study to study. Three studies included patients with bipolar disorder whose results could not be separated. These studies were included as the number of patients with a diagnosis of bipolar disorder formed less than $20 \%$ of the total number in the trial group. 
Most studies used the HRSD (albeit in different versions) as the outcome measure, but some used criteria for remission and others for response. Some studies used psychotic symptoms as part of the response definition, whereas others did not. It was not possible to convert the authors' defined response data into rates based on a single definition (such as a 50\% reduction in HRSD score), so the reviewers used response data as reported by the original authors of the primary studies, with a preference for response of depression (rather than psychosis) where available.

Drop-out rates varied from 9 to $45 \%$ and the reasons for drop-out were not reported in all studies. In addition, those who dropped out were not included in the analysis in all studies. The reviewers recalculated intention-to-treat (ITT) response rates (see Box 1 for an explanation) for 8 of the 12 studies, using all randomly assigned participants as the denominator, regardless of subsequent drop-out.

\section{Main comparisons}

\section{Comparisons with combination (antidepressant plus antipsychotic) treatment}

There were four studies (five comparisons) of combination treatment (antidepressant plus antipsychotic) v. antidepressants alone. Two of the four studies compared a tricyclic antidepressant plus antipsychotic $v$. tricyclics alone. Pooling of the data from these two studies (as in the initial review in 2005) in an ITT analysis did not reveal a difference. One study found superiority of venlafaxine plus quetiapine over venlafaxine alone (but not over imipramine alone) $(n=80$, relative risk (RR) $0.51,95 \%$ CI $0.31-0.83$ ). Pooling the data comparing the combination $v$. antidepressant alone resulted in a difference favouring the combination ( $n=245$, RR 1.42 , 95\% CI 1.11-1.80). Removing the comparisons of a combination with a different antidepressant alone still found a statistically significant difference in favour of the combination ( $n=157$, RR 1.7, 95\% CI 1.19-2.43).

Two studies of combined fluoxetine plus olanzapine showed more efficacy than placebo ( $n=148$, RR $1.86,95 \%$ CI $1.23-2.82)$, with no difference in drop-out rates.

Four studies looked at combination treatment $v$. antipsychotic alone. Amitriptyline plus perphenazine, olanzapine plus fluoxetine, and olanzapine plus sertraline all showed on analysis of each comparison a superior efficacy for the combination over antipsychotic alone. When data from all four studies were combined, this effect continued, favouring the combination $(n=447, \mathrm{RR} 1.83$, 95\% CI 1.40-2.38).

\section{BOX 1 Intention-to-treat analysis}

The results of randomised controlled trials (RCTs) can be biased if the data analysed are from comparison groups that differ from the groups originally generated by randomisation.

This might be because participants drop out of the study, do not adhere fully to the therapy or are switched to alternative therapies.

Effect estimates can be biased if these data are either left out of the analysis or allocated to a different comparison group. For example, an efficacy estimate might be inflated or adverse effects not accounted for. Intention-to-treat (ITT) is the least biased way to estimate intervention effects in RCTs.

The principles of ITT analysis are:

- participants are kept in the groups to which they were randomised, regardless of the intervention they actually received

- outcome data are measured for all participants

- all randomised participants are included in the analysis.

In practice, RCTs are very likely to have missing outcome data, especially if there is a long follow-up period. This can be managed by techniques such as 'last observation carried forward' (LOCF) and using assumed outcomes. Missing data might be replaced with an assumed poor outcome, a mean value or one predicted by a regression analysis.

For further explanation see Higgins \& Green (2011)

\section{Comparisons of antidepressant or antipsychotic as single treatment}

One small study of antidepressant (amitriptyline) alone $(n=27)$ and two studies of antipsychotic (olanzapine) alone $(n=201)$ showed no difference compared with placebo. One RCT compared perphenazine $v$. amitriptyline $(n=36)$, but no difference in efficacy was reported.

\section{Drop-out rates}

In all studies (apart from Meyers et al, 2009) there were no differences in drop-out rates between treatment groups, which could be considered as a proxy measure of acceptability of treatment. (The study by Meyers et al showed a higher rate of dropout among those treated with olanzapine compared with those on olanzapine plus fluoxetine - perhaps because of lack of response in the first group.)

\section{Discussion and clinical applicability}

The review found that the combination of an antidepressant plus an antipsychotic is more effective in psychotic depression than antidepressant monotherapy, antipsychotic monotherapy or 
placebo. The limited evidence available also shows no benefit of antipsychotic alone or antidepressant alone over placebo.

The conclusions are limited, as they are based on only 12 studies, with a small number of antidepressants and antipsychotics used. Thus, it is not clear whether the conclusions can be extrapolated to other medications within the same class. For example, the evidence that the combination of antidepressant and antipsychotic is more effective than antidepressant alone has been obtained only for the combination of venlafaxine and quetiapine v. venlafaxine alone (Wijkstra 2010). The evidence that the combination is more effective than an antipsychotic alone has been obtained in RCTs for two antipsychotics: perphenazine with/without amitriptyline (Spiker 1985); and olanzapine with/ without fluoxetine (Rothschild 2004) and with/ without sertraline (Meyers 2009).

\section{Study quality}

The quality of the primary evidence also has an impact on the confidence with which we can

\section{BOX $2 \mathrm{MeSH}$ terms}

Medical subject headings (MeSH) are a controlled vocabulary (thesaurus) of predefined terms.

They cover all aspects of medicine and healthcare

They are used to index journal articles and books, and therefore facilitate searching.

They were created and are updated by the US National Library of Medicine (NLM), and are used by Medline and PubMed, as well as the Cochrane Library and ClinicalTrials.gov

Instead of allowing numerous variations in words and phrases, they seek to 'control' the vocabulary by grouping related concepts under a single preferred term.

Thus, all indexers use the same standard terms to describe a subject area, regardless of the term the author has chosen to use. For example, all items relating to heart attacks are indexed as 'myocardial infarction', whether or not the author has actually used this term.

As important new concepts appear, a new $\mathrm{MeSH}$ term is created.

When searching, you can 'explode' the search to retrieve all documents relating to distal subject terms, or 'focus' your search to choose citations that have the subject as the major component. view the results. Most studies were relatively small, reflecting the difficulties of conducting RCTs in this population, and the methodology was variable. For example, in the older studies, concealment of allocation was assumed, but not explicitly described. The majority of participants were in-patients, reflecting the severity of their illness. Furthermore, those who took part in the studies may not represent the full range of patients with psychotic depression. Those who were the most severely ill may not have been able or willing to give their consent because of their psychotic ideation, or may have needed urgent treatment with all medications available (i.e. antidepressant and antipsychotic), thus excluding them from entering a randomised trial, or may have needed ECT on an urgent basis.

\section{Diagnosis}

There is also the issue of diagnostic assessment. Some of the psychotic features of psychotic depression can be difficult to distinguish from the thoughts and beliefs often present in severe depression without psychotic features. For example, the boundary separating depressive thoughts of guilt or low self-esteem which are common in nonpsychotic depression from psychotic delusional beliefs of guilt can vary between assessing clinicians, even when using standardised criteria. In clinical practice, psychotic depression can be missed because psychotic symptoms are subtle, intermittent or concealed by the patient (Rothschild 2008).

\section{Outcome measures}

The primary studies used different measures of response and it was not possible to recalculate these using a single definition, so the review authors relied on response rates as defined by the primary authors. In addition, in some studies, response definition included response with regard to psychotic symptoms. Although clinically relevant, this meant that there was some variability in the definitions used, which was only partially addressed by the review preferring response in depressive symptoms where available.

\section{Search terms}

One of the difficulties encountered by the reviewers in their search for primary studies was that there is no keyword (or MeSH term - see Box 2) for psychotic depression. Therefore, the reviewers had to search all RCTs of depression to see whether they included participants with psychotic features, and whether such participants were reported as a separate subgroup. Because the search was 
more difficult, there is always the possibility that some data may have been missed, although the reviewers made every effort by searching all RCTs in depression as a whole. In three studies, the subgroup of participants with psychotic depression was part of a greater group of participants with psychotic and non-psychotic depression, although the subgroups were not stratified before random assignment.

\section{Results}

Despite the difficulties, the review provides the best summary of the data to date. Importantly, the inclusion criteria were strict and only RCTs were included. Also, all the data were reanalysed where necessary so that only ITT data were included, minimising any bias arising from different drop-out rates. The conclusions of this 2015 Cochrane review differ from those of the previous version (Wijkstra 2005). Although agreeing with the current review that treatment with an antipsychotic alone is not a good option, the 2005 review had also concluded that either the combination of an antidepressant and an antipsychotic or starting an antidepressant alone and adding an antipsychotic if the patient does not respond, appeared to be an appropriate option for patients with psychotic depression. The two large studies - Meyers et al (2009) with 259 participants, and Wijkstra et al (2010) with 122 participants - added in the current review have changed the conclusions of the analyses.

The current review is in line with another recent meta-analysis (Farahani 2012), which also concluded that combination treatment is more effective than monotherapy, with no differences in drop-out rates. This analysis used a slightly different set of studies as its inclusion criteria were different, but the conclusion was essentially the same. Current guidelines are also in line with this conclusion, with the American Psychiatric Association (2010), NICE (2009) and the British Association of Psychopharmacology (Cleare 2015) all recommending combination antidepressant plus antipsychotic treatment as first-line pharmacological treatment in psychotic depression.

\section{Conclusions}

Psychotic depression is a severe and not uncommon condition, but it is an area with little research, perhaps because of the difficulties of conducting high-quality trials among affected patients. This review gives clear evidence that combination treatment should be used as first-line therapy where pharmacological treatment is considered. However, it was not able to answer some key questions. These include assessing the relative merits of antipsychotics and/or antidepressants $v$. ECT or ketamine in acute treatment, and which treatments are best for long-term maintenance and prevention of recurrence. Regarding the latter question, there has been little evidence to date of the best pharmacological treatment in the longer term, although this is now being investigated in a clinical trial by the STOP-PD II study group (trial number NCT01427608; Flint 2013). This extends the original STOP-PD study (Meyers 2009), which established that the combination of olanzapine and sertraline was significantly better than olanzapine alone in achieving remission. This continuation study will compare the combination with sertraline alone in maintaining remission over a 36-month follow-up and is due to be completed later in 2017. It is hoped that these data will extend our knowledge of the ongoing management of this severe and important condition.

\section{Acknowledgements}

The author acknowledges support from the NIHR Oxford cognitive health Clinical Research Facility and thanks Andrea Cipriani, Associate Professor at Oxford University Department of Psychiatry, for his comments and suggestions. The views expressed are those of the author and not necessarily those of the NHS, the NIHR or the Department of Health.

\section{References}

American Psychiatric Association (2010) Practice Guideline for the Treatment of Patients with Major Depressive Disorder (Revision). APA.

Cleare A, Pariante CM, Young AH, et al (2015) Evidence-based guidelines for treating depressive disorders with antidepressants: a revision of the 2008 British Association for Psychopharmacology guidelines. Journal of Psychopharmacology, 29: 459-25

Coryell W, Pfohl B, Zimmerman M (1984) The clinical and neuroendocrine features of psychotic depression. Journal of Nervous and Mental Disease, 172: $521-8$.

Coryell W (1998) The treatment of psychotic depression. Journal of Clinical Psychiatry, 59 (suppl 1): 22-9.

Farahani A, Correll CU (2012) Are antipsychotics or antidepressants needed for psychotic depression? A systematic review and meta-analysis of trials comparing antidepressant or antipsychotic monotherapy with combination treatment. Journal of Clinical Psychiatry, 73: 486-96.

Flint AJ, Meyers BS, Rothschild AJ, et al (2013) Sustaining remission of psychotic depression: rationale, design and methodology of STOP-PD II. BMC Psychiatry, 13: 38.

Higgins JPT, Green S (eds) (2011) Cochrane Handbook for Systematic Reviews of Interventions: Version 5.1.0 (updated March 2011). Cochrane Collaboration.

Johnson J, Horwath E, Weisman MM (1991) The validity of major depression with psychotic features based on a community study. Archives of General Psychiatry, 48: 1075-81.

Kessler RC, Berglund P, Demler O, et al (2003) The epidemiology of major depressive disorder: results from the National Comorbidity Survey Replication (NCS-R). JAMA, 289: 3095-105. 
Meyers BS, Flint AJ, Rothschild AJ, et al (2009) A double-blind randomized controlled trial of olanzapine plus sertraline vs olanzapine plus placebo for psychotic depression: the study of pharmacotherapy of psychotic depression (STOP-PD). Archives of General Psychiatry, 66: 838-47.

National Institute for Health and Care Excellence (2009) Depression in Adults: Recognition and Management (Updated 2016) (Clinical Guideline CG90). NICE.

Ohayon MM, Schatzberg AF (2002) Prevalence of depressive episodes with psychotic features in the general population. American Journal of Psychiatry, 159: 1855-61.

Parker G, Roy K, Hadzi-Pavlovic D, et al (1992) Psychotic (delusional) depression: a meta-analysis of physical treatments. Journal of Affective Disorders, 24: 17-24.

Rothschild AJ (2003) Challenges in the treatment of depression with psychotic features. Biological Psychiatry, 53: 680-90.

Rothschild AJ, Williamson DJ, Tohen MF, et al (2004) A double-blind, randomized study of olanzapine and olanzapine/fluoxetine combination for major depression with psychotic features. Journal of Clinical Psychopharmacology, 24: 365-73.
Rothschild AJ, Winer J, Flint AJ, et al (2008) Missed diagnosis of psychotic depression at 4 academic medical centers. Journal of Clinical Psychiatry, 69: 1293-6.

Spiker DG, Weiss JC, Dealy RS, et al (1985) The pharmacological treatment of delusional depression. American Journal of Psychiatry, 142: 430-6.

Wijkstra J, Lijmer J, Balk F, et al (2005) Pharmacological treatment for psychotic depression. Cochrane Database of Systematic Reviews, 4: CD004044.

Wijkstra J, Schubart CD, Nolen WA (2007) Treatment of unipolar psychotic depression: the use of evidence in practice guideline. World Journal of Biological Psychiatry, 24: 1-7

Wijkstra J, Burger H, van den Broek WW, et al (2010) Treatment of unipolar psychotic depression: a randomized, double-blind study comparing imipramine, venlafaxine, and venlafaxine plus quetiapine. Acta Psychiatrica Scandinavica, 121: 190-200.

Wijkstra J, Lijmer J, Burger H, et al (2015) Pharmacological treatment for psychotic depression. Cochrane Database of Systematic Reviews, 7: CD004044 